\title{
A HOPF ALGEBRA ASSOCIATED TO A LIE PAIR
}

\author{
ZHUO CHEN, MATHIEU STIÉNON, AND PING XU
}

In tribute to Alan Weinstein on the occasion of his seventieth birthday

\begin{abstract}
The quotient $L / A[-1]$ of a pair $A \hookrightarrow L$ of Lie algebroids is a Lie algebra object in the derived category $D^{b}(\mathcal{A})$ of the category $\mathcal{A}$ of left $\mathcal{U}(A)$-modules, the Atiyah class $\alpha_{L / A}$ being its Lie bracket. In this note, we describe the universal enveloping algebra of the Lie algebra object $L / A[-1]$ and we prove that it is a Hopf algebra object in $D^{b}(\mathcal{A})$.
\end{abstract}

\section{INTRODUCTION}

Let $A$ be a Lie algebroid over a manifold $M$. Its space of smooth sections $\Gamma(A)$ is a Lie-Rinehart algebra over the commutative ring $R=C^{\infty}(M)$. By an $A$-module, we mean a module over the Lie-Rinehart algebra corresponding to the Lie algebroid $A$, i.e. a module over the associative algebra $\mathcal{U}(A)$.

Recall that the universal enveloping algebra $\mathcal{U}(A)$ of a Lie algebroid $A$ over $M$ is simultaneously an associative algebra and an $R$-bimodule. In case the Lie algebroid $A$ is real, $\mathcal{U}(A)$ is canonically identified to the algebra of left-invariant s-fiberwise differential operators on the local Lie groupoid $\mathscr{A}$ integrating $A$. Let us recall its construction.

The vector space $\mathfrak{g}=R \oplus \Gamma(A)$ admits a natural Lie algebra structure given by the Lie bracket

$$
[f+X, g+Y]=\rho(X) g-\rho(Y) f+[X, Y]
$$

where $f, g \in R$ and $X, Y \in \Gamma(A)$. Here $\rho$ denotes the anchor map. Let $i$ denote the natural inclusion of $\mathfrak{g}$ into its universal enveloping algebra $\mathcal{U}(\mathfrak{g})$. The universal enveloping algebra $\mathcal{U}(A)$ of the Lie algebroid $A$ is the quotient of the subalgebra of $\mathcal{U}(\mathfrak{g})$ generated by $i(\mathfrak{g})$ by the two-sided ideal generated by the elements of the form $i(f) \otimes i(g+Y)-i(f g+f Y)$ with $f, g \in R$ and $Y \in \Gamma(A)$.

When $A$ is a Lie algebra, $\mathcal{U}(A)$ is indeed the usual universal enveloping algebra. On the other hand, when $A$ is the tangent bundle $T M, \mathcal{U}(A)$ is the algebra of differential operators on $M$.

We use the symbol $\mathcal{A}$ to denote the abelian category of $A$-modules. Abusing terminology, we say that a vector bundle $E$ over $M$ is an $A$-module if $\Gamma(E) \in \mathcal{A}$.

Given a Lie pair $(L, A)$ of algebroids, i.e. a Lie algebroid $L$ with a Lie subalgebroid $A$, the Atiyah class $\alpha_{E}$ of an $A$-module $E$ relative to the pair $(L, A)$ is defined as the obstruction to the existence of an $A$-compatible $L$-connection on the vector bundle $E$. An $L$-connection $\nabla$ on an $A$-module $E$ is said to be $A$-compatible if it extends the given flat $A$-connection on $E$ and satisfies $\nabla_{a} \nabla_{l}-\nabla_{l} \nabla_{a}=\nabla_{[a, l]}$ for all $a \in \Gamma(A)$ and $l \in \Gamma(L)$. This fairly recently defined class (see [1]) has as double

Research partially supported by NSF grant DMS1101827, NSA grant H98230-12-1-0234, and NSFC grants 11001146 and 11471179. 
origin, which it generalizes, the Atiyah class of holomorphic vector bundles and the Molino class of foliations.

The quotient $L / A$ of any Lie pair $(L, A)$ is an $A$-module [1]. Its Atiyah class $\alpha_{L / A}$ can be described as follows. Choose an $L$-connection $\nabla$ on $L / A$ extending the $A$-action. Its curvature is the vector bundle map $R^{\nabla}: \wedge^{2} L \rightarrow \operatorname{End}(E)$ defined by $R^{\nabla}\left(l_{1}, l_{2}\right)=\nabla_{l_{1}} \nabla_{l_{2}}-\nabla_{l_{2}} \nabla_{l_{1}}-\nabla_{\left[l_{1}, l_{2}\right]}$, for all $l_{1}, l_{2} \in \Gamma(L)$. Since $L / A$ is an $A$-module, $R^{\nabla}$ vanishes on $\wedge^{2} A$ and, therefore, determines a section $R_{L / A}^{\nabla}$ of $A^{*} \otimes(L / A)^{*} \otimes \operatorname{End}(L / A)$. It was proved in [1] that $R_{L / A}^{\nabla}$ is a 1-cocycle for the Lie algebroid $A$ with values in the $A$-module $(L / A)^{*} \otimes \operatorname{End}(L / A)$ and that its cohomology class $\alpha_{L / A} \in H^{1}\left(A ;(L / A)^{*} \otimes \operatorname{End}(L / A)\right)$ is independent of the choice of the connection.

Let $\mathrm{Ch}^{b}(\mathcal{A})$ denote the category of bounded complexes in $\mathcal{A}$ and let $D^{b}(\mathcal{A})$ denote the corresponding derived category. We write $L / A[-1]$ to denote the quotient $L / A$ regarded as a complex in $\mathcal{A}$ concentrated in degree 1 .

The following was proved in 1 .

Proposition 1.1 ([1]). Let $(L, A)$ be a Lie algebroid pair. The Atiyah class $\alpha_{L / A}$ of the quotient $L / A$ relative to the pair $(L, A)$ determines a morphism

$$
L / A[-1] \otimes L / A[-1] \rightarrow L / A[-1]
$$

in the derived category $D^{b}(\mathcal{A})$ making $L / A[-1]$ a Lie algebra object in $D^{b}(\mathcal{A})$.

It is well known that every ordinary Lie algebra $\mathfrak{g}$ admits a universal enveloping algebra $\mathcal{U}(\mathfrak{g})$, which is a Hopf algebra. We are thus led to the following natural questions: does there exist a universal enveloping algebra for $L / A[-1]$ in $D^{b}(\mathcal{A})$ and, if so, is it a Hopf algebra object?

In this Note, we give a positive answer to the questions above. For a complex manifold $X$, the Atiyah class of the Lie pair $\left(T_{X} \otimes \mathbb{C}, T_{X}^{0,1}\right)$ is simply the usual Atiayh class of the holomorphic tangent bundle $T_{X}$ recently exploited by Kapranov [2]. It was proved that the universal enveloping algebra of the Lie algebra object $T_{X}[-1]$ in $D^{b}(X)$ is the Hochschild cochain complex $\left(\mathcal{D}_{\text {poly }}^{\bullet}(X), d\right)$ [5, 6, 7,. This result played an important role in the study of several aspects of complex geometry including the Riemann-Roch theorem [5], the Chern character [6] and the Rozansky-Witten invariants [7, 8]. Applications of our result will be developed elsewhere.

\section{Hochschild-Kostant-Rosenberg MaP}

It is known [9] that the universal enveloping algebra $\mathcal{U}(L)$ of a Lie algebroid $L$ admits a cocommutative coassociative coproduct $\Delta: \mathcal{U}(L) \rightarrow \mathcal{U}(L) \tilde{\otimes} \mathcal{U}(L)$, which is defined on generators as follows: $\Delta(f)=f \tilde{\otimes} 1=1 \tilde{\otimes} f, \forall f \in R$ and $\Delta(l)=l \tilde{\otimes} 1+1 \tilde{\otimes} l$, $\forall l \in \Gamma(L)$. Here, and in the sequel, $\tilde{\otimes}$ stands for the tensor product of left $R$ modules. Moreover, $\mathcal{U}(L)$ is an $L$-module since each section $l$ of $L$ acts on $\mathcal{U}(L)$ by left multiplication: $\nabla_{l} u=l \cdot u, \forall u \in \mathcal{U}(L)$.

Now, given a Lie pair $(L, A)$, consider the quotient $\mathcal{D}_{\text {poly }}^{1}$ of $\mathcal{U}(L)$ by the left ideal generated by $\Gamma(A)$. It is straighforward to see that the comultiplication on $\mathcal{U}(L)$ induces a comultiplication $\Delta: \mathcal{D}_{\text {poly }}^{1} \rightarrow \mathcal{D}_{\text {poly }}^{1} \tilde{\otimes} \mathcal{D}_{\text {poly }}^{1}$ on $\mathcal{D}_{\text {poly }}^{1}$ and the action of $L$ on $\mathcal{U}(L)$ determines an action of $A$ on $\mathcal{D}_{\text {poly }}^{1}$. 
Lemma 2.1. The quotient $\mathcal{D}_{\text {poly }}^{1}=\frac{\mathcal{U}(L)}{\mathcal{U}(L) \Gamma(A)}$ is simultaneously a cocommutative coassociative $R$-coalgebra and an A-module. Moreover, its comultiplication is compatible with its A-action:

$$
\nabla_{X}(\Delta p)=\Delta\left(\nabla_{X} p\right), \quad \forall X \in \Gamma(A), p \in \mathcal{D}_{\text {poly }}^{1} .
$$

Let $\mathcal{D}_{\text {poly }}^{n}$ denote the $n$-th tensorial power $\mathcal{D}_{\text {poly }}^{1} \tilde{\otimes} \cdots \tilde{\otimes} \mathcal{D}_{\text {poly }}^{1}$ of $\mathcal{D}_{\text {poly }}^{1}$ and, for $n=0$, set $\mathcal{D}_{\text {poly }}^{0}=R$. We define a coboundary operator $d: \mathcal{D}_{\text {poly }}^{\bullet} \rightarrow \mathcal{D}_{\text {poly }}^{\bullet+1}$ on $\mathcal{D}_{\text {poly }}^{\bullet}=\bigoplus_{n=0}^{\infty} \mathcal{D}_{\text {poly }}^{n}$ by

$$
\begin{aligned}
d\left(p_{1} \tilde{\otimes} \cdots \tilde{\otimes} p_{n}\right)= & 1 \tilde{\otimes} p_{1} \tilde{\otimes} \cdots \tilde{\otimes} p_{n}-\left(\Delta p_{1}\right) \tilde{\otimes} \cdots \tilde{\otimes} p_{n}+p_{1} \tilde{\otimes}\left(\Delta p_{2}\right) \tilde{\otimes} \cdots \tilde{\otimes} p_{n}-\cdots \\
& +(-1)^{n} p_{1} \tilde{\otimes} \cdots \tilde{\otimes} p_{n-1} \tilde{\otimes}\left(\Delta p_{n}\right)+(-1)^{n+1} p_{1} \tilde{\otimes} \cdots \tilde{\otimes} p_{n} \tilde{\otimes} 1
\end{aligned}
$$

for any $p_{1}, p_{2}, \ldots, p_{n} \in \mathcal{D}_{\text {poly }}^{1}$. Since the comultiplication $\Delta$ is compatible with the action of $A$, the operator $d$ is a morphism of $A$-modules. Moreover, $\Delta$ being coassociative, $d$ satisfies $d^{2}=0$. Thus $\left(\mathcal{D}_{\text {poly }}^{\bullet}, d\right)$ is an object of $\mathrm{Ch}^{b}(\mathcal{A})$.

When endowed with the trivial coboundary operator, the space of sections of

$$
S^{\bullet}(L / A[-1])=\bigoplus_{k=0}^{\infty} S^{k}(L / A[-1])=\bigoplus_{k=0}^{\infty}\left(\wedge^{k} L / A\right)[-k]
$$

is a complex of $A$-modules:

$$
0 \rightarrow R \stackrel{0}{\rightarrow} \Gamma(L / A) \stackrel{0}{\rightarrow} \Gamma\left(\wedge^{2}(L / A)\right) \stackrel{0}{\rightarrow} \Gamma\left(\wedge^{3}(L / A)\right) \stackrel{0}{\rightarrow} \cdots
$$

The natural inclusion $\Gamma(L / A) \hookrightarrow \mathcal{D}_{\text {poly }}^{1}$ extends naturally to the Hochschild-KostantRosenberg map

$$
\operatorname{HKR}: \Gamma\left(S^{\bullet}(L / A[-1])\right) \rightarrow \mathcal{D}_{\text {poly }}^{\bullet}
$$

by skew-symmetrization:

$$
\begin{aligned}
& \operatorname{HKR}\left(b_{1} \wedge \cdots \wedge b_{n}\right)=\frac{1}{n !} \sum_{\sigma \in S_{n}} \operatorname{sgn}(\sigma) b_{\sigma(1)} \tilde{\otimes} b_{\sigma(2)} \tilde{\otimes} \cdots \tilde{\otimes} b_{\sigma(n)}, \\
& \forall b_{1}, \cdots, b_{n} \in \Gamma(L / A) .
\end{aligned}
$$

Proposition 2.2. In $\mathrm{Ch}^{b}(\mathcal{A})$, the Hochschild-Kostant-Rosenberg map is a quasiisomorphism from $\left(\Gamma\left(S^{\bullet}(L / A[-1])\right), 0\right)$ to $\left(\mathcal{D}_{\text {poly }}^{\bullet}, d\right)$.

Sketch of proof. Assuming $L$ and $A$ are real Lie algebroids, let $\mathscr{L}$ and $\mathscr{A}$ be local Lie groupoids integrating $L$ and $A$ respectively. The source map $s: \mathscr{L} \rightarrow M$ induces a surjective submersion $J: \mathscr{L} / \mathscr{A} \rightarrow M$. The right quotient $\mathscr{L} / \mathscr{A}$ is a left $\mathscr{L}$ homogeneous space with momentum map $J[4$. Therefore, it admits an infinitesimal $L$-action, and hence an infinitesimal $A$-action. The coalgebra $\mathcal{D}_{\text {poly }}^{1}$ may be regarded as the space of distributions on the $J$-fibers of $\mathscr{L} / \mathscr{A}$ supported on $M$. Its $A$-module structure then stems from the infinitesimal $A$-action on $\mathscr{L} / \mathscr{A}$. The $n$-th tensorial power $\mathcal{D}_{\text {poly }}^{n}$ may be viewed as the space of $n$-differential operators on the $J$-fibers of $\mathscr{L} / \mathscr{A}$ evaluated along $M$ and the differential $d$ as the Hochschild coboundary. The conclusion follows from the classical Hochschild-Kostant-Rosenberg theorem. To prove the proposition for complex Lie algebroids, it suffices to consider formal groupoids instead of local Lie groupoids [3]. 


\section{Universal enveloping algebra of $L / A[-1]$ IN $D^{b}(\mathcal{A})$}

Following Markarian [5], Ramadoss [6], and Roberts-Willerton [7], we introduce the following:

Definition 3.1. If it exists, the universal enveloping algebra of a Lie algebra object $\mathcal{G}$ in $D^{b}(\mathcal{A})$ is an associative algebra object $\mathcal{H}$ in $D^{b}(\mathcal{A})$ together with a morphism of Lie algebras $i: \mathcal{G} \rightarrow \mathcal{H}$ satisfying the following universal property: given any associative algebra object $\mathcal{K}$ and any morphism of Lie algebras $f: \mathcal{G} \rightarrow \mathcal{K}$ in $D^{b}(\mathcal{A})$, there exists a unique morphism of associative algebras $f^{\prime}: \mathcal{H} \rightarrow \mathcal{K}$ in $D^{b}(\mathcal{A})$ such that $f=f^{\prime} \circ i$.

In view of the similarity between $\left(\mathcal{D}_{\text {poly }}^{\bullet}, d\right)$ and the Hochschild cochain complex, we define a cup product $\cup$ on $\mathcal{D}_{\text {poly }}^{\bullet}$ by setting $P \cup Q=P \tilde{\otimes} Q$, for all $P, Q \in \mathcal{D}_{\text {poly }}^{\bullet}$. Is is simple to check that

$$
d(P \cup Q)=d P \cup Q+(-1)^{|P|} P \cup d Q,
$$

for all homogeneous $P, Q \in \mathcal{D}_{\text {poly }}^{\bullet}$.

Proposition 3.2. For any Lie pair $(L, A)$ of algebroids, $\left(\mathcal{D}_{\text {poly }}^{\bullet}, d, \cup\right)$ is an associative algebra object in $D^{b}(\mathcal{A})$, which is in fact the universal enveloping algebra of the Lie algebra $L / A[-1]$ in $D^{b}(\mathcal{A})$.

Consider the inclusion $\eta: R \hookrightarrow \mathcal{D}_{\text {poly }}^{n}$, the projection $\varepsilon: \mathcal{D}_{\text {poly }}^{n} \rightarrow R$, and the maps $t: \mathcal{D}_{\text {poly }}^{\bullet} \rightarrow \mathcal{D}_{\text {poly }}^{\bullet}$ and $\tilde{\Delta}: \mathcal{D}_{\text {poly }}^{\bullet} \rightarrow \mathcal{D}_{\text {poly }}^{\bullet} \underset{R}{\otimes} \mathcal{D}_{\text {poly }}^{\bullet}$ defined, respectively, by

$$
t\left(p_{1} \tilde{\otimes} p_{2} \tilde{\otimes} \cdots \tilde{\otimes} p_{n}\right)=(-1)^{\frac{n(n-1)}{2}} p_{n} \tilde{\otimes} p_{n-1} \tilde{\otimes} \cdots \tilde{\otimes} p_{1}
$$

and

$\tilde{\Delta}\left(p_{1} \tilde{\otimes} p_{2} \tilde{\otimes} \cdots \tilde{\otimes} p_{n}\right)=\sum_{i+j=n} \sum_{\sigma \in \mathfrak{S}_{i}^{j}} \operatorname{sgn}(\sigma)\left(p_{\sigma(1)} \tilde{\otimes} \cdots \tilde{\otimes} p_{\sigma(i)}\right) \otimes\left(p_{\sigma(i+1)} \tilde{\otimes} \cdots \tilde{\otimes} p_{\sigma(n)}\right)$, where $\mathfrak{S}_{i}^{j}$ denotes the set of $(i, j)$-shuffles ${ }^{1}$

Theorem 3.3. For any Lie pair $(L, A)$ of algebroids, $\left(\mathcal{D}_{\text {poly }}^{\bullet}, d\right)$ with the multiplication $\cup$, the comultiplication $\tilde{\Delta}$, the unit $\eta$, the counit $\varepsilon$, and the antipode $t$, is a Hopf algebra object in $D^{b}(\mathcal{A})$.

\section{RAmadoss's APPROACH: $L\left(\mathcal{D}_{\text {poly }}^{1}\right)$}

To prove Proposition 3.2 and Theorem 3.3 we essentially follow Ramadoss's approach [6]. Let $L\left(\mathcal{D}_{\text {poly }}^{1}\right)$ be the (graded) free Lie algebra generated over $R$ by $\mathcal{D}_{\text {poly }}^{1}$ concentrated in degree 1 . In other words, $L\left(\mathcal{D}_{\text {poly }}^{1}\right)$ is the smallest Lie subalgebra of $\mathcal{D}_{\text {poly }}^{\bullet}$ containing $\mathcal{D}_{\text {poly }}^{1}$. The Lie bracket of two vectors $u \in \mathcal{D}_{\text {poly }}^{i}$ and $v \in \mathcal{D}_{\text {poly }}^{j}$ is the vector $[u, v]=u \tilde{\otimes} v-(-1)^{i j} v \tilde{\otimes} u \in \mathcal{D}_{\text {poly }}^{i+j}$. Actually, $L\left(\mathcal{D}_{\text {poly }}^{1}\right)$ is made of all linear combinations of elements of the form $\left[p_{1},\left[p_{2},\left[\cdots,\left[p_{n-1}, p_{n}\right] \cdots\right]\right]\right]$ with $p_{1}, \ldots, p_{n} \in \mathcal{D}_{\text {poly }}^{1}$. One checks that $L\left(\mathcal{D}_{\text {poly }}^{1}\right)$ is a $d$-stable $A$-submodule of $\mathcal{D}_{\text {poly }}^{\bullet}$ and that its Lie bracket is a chain map with respect to the coboundary operator $d$. Therefore $\left(L\left(\mathcal{D}_{\text {poly }}^{1}\right), d\right)$ is a Lie algebra object in $\operatorname{Ch}^{b}(\mathcal{A})$.

\footnotetext{
${ }^{1} \mathrm{An}(i, j)$-shuffle is a permutation $\sigma$ of the set $\{1,2, \cdots, i+j\}$ such that $\sigma(1) \leq \sigma(2) \leq \cdots \leq$ $\sigma(i)$ and $\sigma(i+1) \leq \sigma(i+2) \leq \cdots \leq \sigma(i+j)$.
} 
Let $S^{\bullet}\left(L\left(\mathcal{D}_{\text {poly }}^{1}\right)\right)$ be the symmetric algebra of $L\left(\mathcal{D}_{\text {poly }}^{1}\right)$ and let

$$
I: S^{\bullet}\left(L\left(\mathcal{D}_{\text {poly }}^{1}\right)\right) \rightarrow \mathcal{D}_{\text {poly }}^{\bullet}
$$

be the symmetrization map:

$$
I\left(z_{1} \odot \cdots \odot z_{n}\right)=\frac{1}{n !} \sum_{\sigma \in S_{n}} \operatorname{sgn}\left(\sigma ; z_{1}, \cdots, z_{n}\right) z_{\sigma(1)} \tilde{\otimes} z_{\sigma(2)} \tilde{\otimes} \cdots \tilde{\otimes} z_{\sigma(n)} .
$$

The Koszul sign $\operatorname{sgn}\left(\sigma ; z_{1}, \cdots, z_{n}\right)$ of a permutation $\sigma$ of the (homogeneous) vectors $z_{1}, z_{2}, \ldots, z_{n} \in S^{\bullet}\left(L\left(\mathcal{D}_{\text {poly }}^{1}\right)\right)$ is determined by the relation

$$
z_{\sigma(1)} \odot z_{\sigma(2)} \odot \cdots \odot z_{\sigma(n)}=\operatorname{sgn}\left(\sigma ; z_{1}, \cdots, z_{n}\right) z_{1} \odot z_{2} \odot \cdots \odot z_{n} .
$$

Lemma 4.1. The symmetrization $I: S^{\bullet}\left(L\left(\mathcal{D}_{\text {poly }}^{1}\right)\right) \rightarrow \mathcal{D}_{\text {poly }}^{\bullet}$ is an isomorphism in $\mathrm{Ch}^{b}(\mathcal{A})$.

Using Lemma 4.1 and the HKR quasi-isomorphism, one can prove that the composition $\beta: \Gamma(L / A[-1]) \rightarrow L\left(\mathcal{D}_{\text {poly }}^{1}\right)$ of the inclusions

$$
\Gamma(L / A[-1]) \subset \mathcal{D}_{\text {poly }}^{1} \subset L\left(\mathcal{D}_{\text {poly }}^{1}\right)
$$

is a quasi-isomorphism in $\mathrm{Ch}^{b}(\mathcal{A})$, which intertwines the Lie brackets on $\Gamma(L / A[-1])$ and $L\left(\mathcal{D}_{\text {poly }}^{1}\right)$.

Proposition 4.2. (1) The inclusion $\beta: \Gamma(L / A[-1]) \rightarrow L\left(\mathcal{D}_{\text {poly }}^{1}\right)$ is a quasiisomorphism in $\mathrm{Ch}^{b}(\mathcal{A})$.

(2) The inclusion $\beta: \Gamma(L / A[-1]) \rightarrow L\left(\mathcal{D}_{\text {poly }}^{1}\right)$ is an isomorphism of Lie algebra objects in $D^{b}(\mathcal{A})$ as the diagram

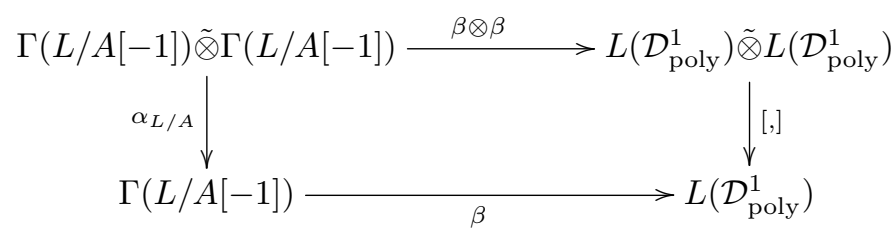

commutes in $D^{b}(\mathcal{A})$.

Proposition 3.2 and Theorem 3.3 now follow immediately.

\section{REFERENCES}

1. Zhuo Chen, Mathieu Stiénon, and Ping Xu, From Atiyah Classes to Homotopy Leibniz Algebras, arXiv: 1204.1075 (2012).

2. M. Kapranov, Rozansky-Witten invariants via Atiyah classes, Compositio Math. 115 (1999), no. 1, 71-113. MR 1671737 (2000h:57056)

3. Niels Kowalzig and Hessel Posthuma, The cyclic theory of Hopf algebroids, J. Noncommut. Geom. 5 (2011), no. 3, 423-476. MR 2817646 (2012f:16081)

4. Zhang-Ju Liu, Alan Weinstein, and Ping Xu, Dirac structures and Poisson homogeneous spaces, Comm. Math. Phys. 192 (1998), no. 1, 121-144. MR 1612164 (99g:58053)

5. Nikita Markarian, The Atiyah class, Hochschild cohomology and the Riemann-Roch theorem, J. Lond. Math. Soc. (2) 79 (2009), no. 1, 129-143. MR 2472137 (2010d:14020)

6. Ajay C. Ramadoss, The big Chern classes and the Chern character, Internat. J. Math. 19 (2008), no. 6, 699-746. MR 2431634 (2010h:14028)

7. Justin Roberts and Simon Willerton, On the Rozansky-Witten weight systems, Algebr. Geom. Topol. 10 (2010), no. 3, 1455-1519. MR 2661534

8. L. Rozansky and E. Witten, Hyper-Kähler geometry and invariants of three-manifolds, Selecta Math. (N.S.) 3 (1997), no. 3, 401-458. MR 1481135 (98m:57041) 
9. Ping Xu, Quantum groupoids, Comm. Math. Phys. 216 (2001), no. 3, 539-581. MR 1815717 (2002f:17033)

Department of Mathematics, Tsinghua University, China

E-mail address: zchen@math.tsinghua.edu.cn

Department of Mathematics, Penn State University, United States

E-mail address: stienon@psu.edu

Department of Mathematics, Penn State University, United States

E-mail address: ping@math.psu.edu 